\title{
Some thoughts on the use of InSAR data to constrain models of surface deformation: Noise structure and data downsampling
}

\author{
Rowena B. Lohman \\ Seismological Laboratory, California Institute of Technology, Pasadena, California 91125, USA \\ Now at Geology and Geophysics, Woods Hole Oceanographic Institution, MS 24, Woods Hole, MA 02543-1050, USA \\ (rlohman@whoi.edu) \\ Mark Simons \\ Seismological Laboratory, California Institute of Technology, Pasadena, California 91125, USA
}

[1] Repeat-pass Interferometric Synthetic Aperture Radar (InSAR) provides spatially dense maps of surface deformation with potentially tens of millions of data points. Here we estimate the actual covariance structure of noise in InSAR data. We compare the results for several independent interferograms with a large ensemble of GPS observations of tropospheric delay and discuss how the common approaches used during processing of InSAR data affects the inferred covariance structure. Motivated by computational concerns associated with numerical modeling of deformation sources, we then combine the datacovariance information with the inherent resolution of an assumed source model to develop an efficient algorithm for spatially variable data resampling (or averaging). We illustrate these technical developments with two earthquake scenarios at different ends of the earthquake magnitude spectrum. For the larger events, our goal is to invert for the coseismic fault slip distribution. For smaller events, we infer the hypocenter location and moment. We compare the results of inversions using several different resampling algorithms, and we assess the importance of using the full noise covariance matrix.

Components: 5784 words, 10 figures.

Keywords: correlated noise; InSAR; Little Skull Mountain.

Index Terms: 1240 Geodesy and Gravity: Satellite geodesy: results (6929, 7215, 7230, 7240); 1241 Geodesy and Gravity: Satellite geodesy: technical issues (6994, 7969); 1209 Geodesy and Gravity: Tectonic deformation (6924).

Received 11 September 2004; Revised 24 November 2004; Accepted 16 December 2004; Published 25 January 2005.

Lohman, R. B., and M. Simons (2005), Some thoughts on the use of InSAR data to constrain models of surface deformation: Noise structure and data downsampling, Geochem. Geophys. Geosyst., 6, Q01007, doi:10.1029/2004GC000841.

\section{Introduction}

[2] The continually improving spatial and temporal coverage of geodetic images such as those provided by Interferometric Synthetic Aperture Radar (InSAR) motivates us to better quantify the sources of error in these data sets in order to rigorously characterize how well we can resolve the deformation sources in space and their evolu- tion in time. Typical deformation sources include magma chambers, dike intrusions, and faults that slip during coseismic, postseismic, and interseismic intervals. The large volume of available observations presents us with a number of challenges, including the characterization and treatment of data noise and practical considerations of how to minimize the computational cost of examining such large data sets. In this paper we explore these two 


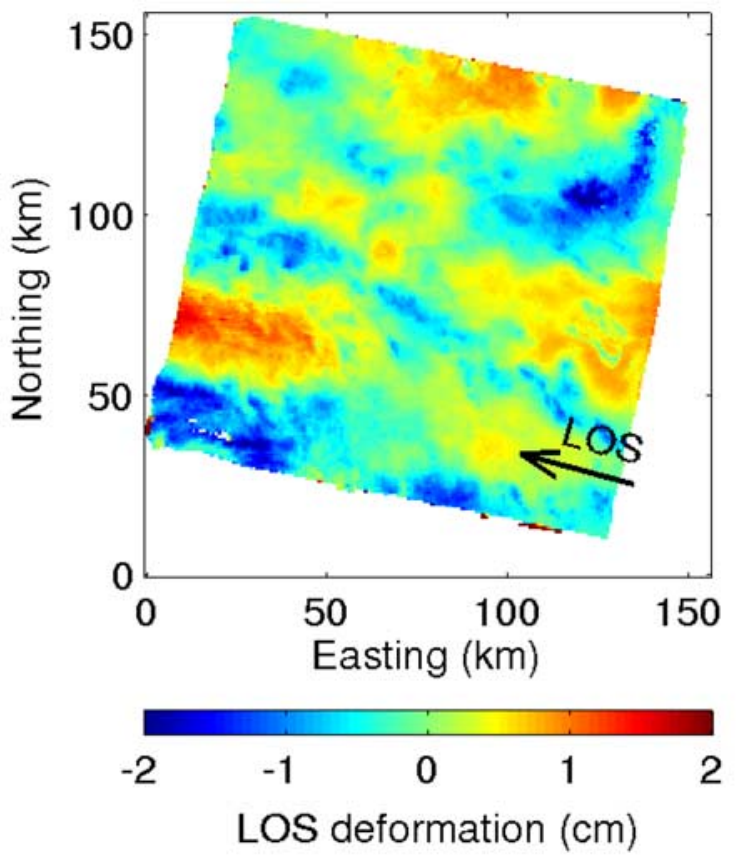

Figure 1. Sample interferogram from the Mojave desert in Southern California, illustrating the spatial covariance of atmospheric noise. Interferogram covers Track 127, Frame 2925, spanning 10 October 1995 to 19 December 1995, with perpendicular baseline $\left(B_{\perp}\right)=$ $37 \mathrm{~m}$. Arrow indicates the horizontal projection of the satellite-to-ground LOS vector.

aspects of the treatment of InSAR data with specific examples of applications to inversions for subsurface fault slip and earthquake location.

[3] First, we present a technique for estimating the covariance structure of noise in InSAR data. To properly combine InSAR and Global Positioning System (GPS) data, and to assess the uncertainties on inferred model parameters, we require an understanding of the character of InSAR data noise. We compare results derived from an ensemble of interferograms with the noise covariance structure derived from GPS data from Southern California [Emardson et al., 2003]. Second, driven by a desire to minimize the computational burden involved in our studies, we demonstrate how we reduce the number of data points used as input to our inversions. Whereas researchers working with earlier, sparser data sets needed to use all the available observations in order to extract as much information as possible about the system of interest [e.g., Harris and Segall, 1987; Segall and Harris, 1987], we now have the luxury of acquiring nearly spatially continuous measurements of ground deformation. Practical considerations, such as the frequent need to perform Monte Carlo tests requir- ing the calculation of a large number of forward models, lead us to develop methods of spatial sampling and averaging of interferograms in a manner that reduces the number of data points while retaining as much information as possible [e.g., Jónsson et al., 2002; Simons et al., 2002].

[4] We end with demonstrations of the application of these techniques on two earthquakes where the goals of our inversions are quite different. For large earthquakes $\left(>\mathrm{M}_{w} 7\right)$ we typically perform linear inversions for the distribution of coseismic fault slip on a plane fixed by the mapped surface trace and aftershock distributions. We use the correct covariance matrices for the noise estimated directly from the data, and illustrate how our resampling algorithm performs compared with previous resampling techniques. For smaller events $\left(<\mathrm{M}_{w} 6\right)$, our goal is often simply to constrain the location of the fault plane, earthquake mechanism, and moment. We perform nonlinear inversions for the best-fitting fault plane, using the Neighborhood Algorithm [e.g., Sambridge, 1998; Lohman et al., 2002]. We demonstrate how neglecting the off-diagonal components of the noise covariance matrix affects the error bounds that we place on our hypocenter estimate.

\section{Estimation and Analysis of the Full Data Covariance}

[5] Satellite-based radar interferograms contain spatially correlated signals (e.g., Figure 1), primarily attributable to atmospheric and ionospheric structures that are commonly coherent over length scales of tens to hundreds of kilometers [e.g., Hanssen, 2001]. Variations in atmospheric water vapor with elevation can also introduce satellite line-of-sight (LOS) delays that are correlated with topography [e.g., Goldstein, 1995; Zebker et al., 1997; Williams et al., 1998; Hanssen, 2001; Jónsson, 2002; Emardson et al., 2003]. In the context of geodetic applications, these signals are treated as measurement noise. In order to assess the significance of features of a source model inferred from InSAR data, we must estimate confidence intervals and model parameter covariances. The degree to which the data noise is correlated affects both these quantities [e.g., Menke, 1989].

[6] In this section, we characterize the structure of noise in interferograms and compare interferograms in Southern California with independent observations of atmospheric delay from GPS sites in the region. We also discuss how the limited 

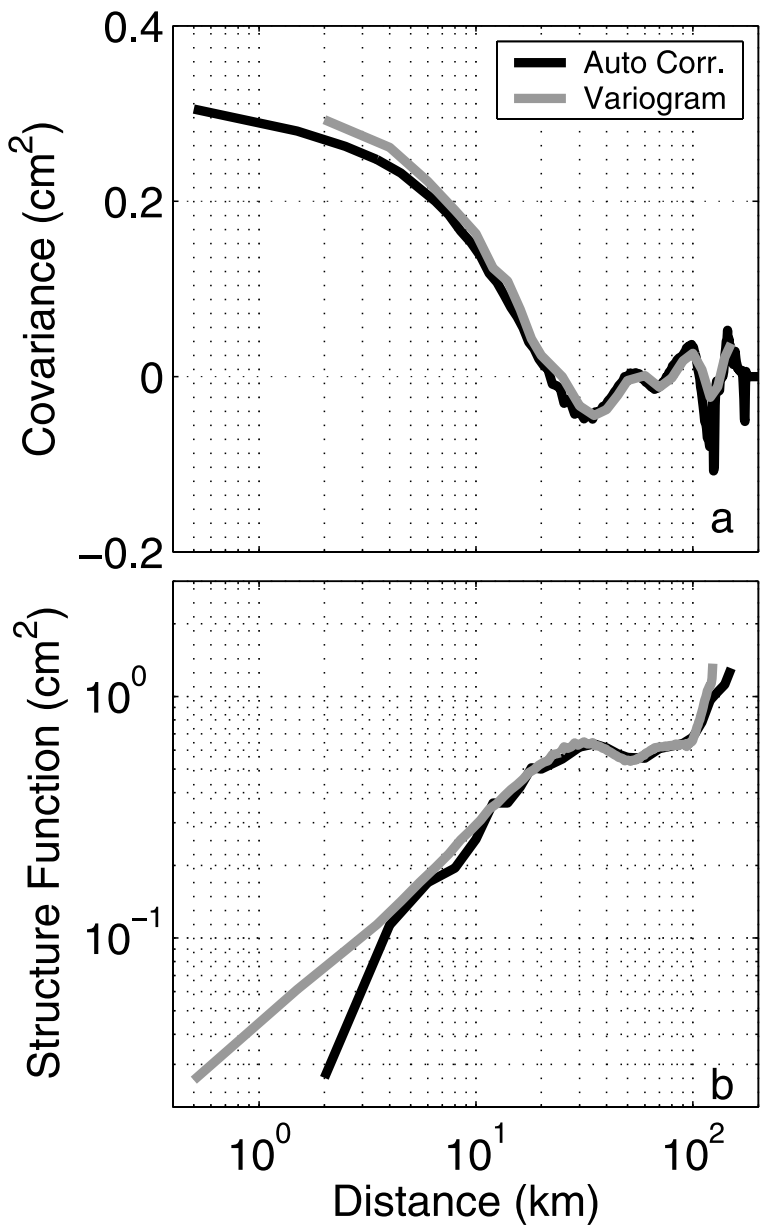

Figure 2. Covariance and structure function versus distance for the interferogram in Figure 1. (a) Covariance, $C(r)$, determined from autocorrelation matrix (black) and by variogram (gray). (b) Structure function, $S(r)$, determined using all pairs of points (black) and from variogram (gray). Note that for most distances the variogram agrees with the result from the full data set.

spatial aperture of SAR images, and our imperfect knowledge of satellite positions, conspire to limit the degree to which we can constrain the true noise covariance.

[7] To estimate the noise covariance matrix, $\mathbf{C}_{\mathbf{n}}$, for individual interferometric pairs, we assume that the noise is spatially stationary and isotropic, i.e., that the covariance between any two points depends only on the distance between them and not on location or on the azimuth between the two points. We define the covariance between two points separated by a scalar distance, $r$, as

$$
\begin{aligned}
C_{n}(r) & =\operatorname{Cov}(\mathbf{x}, \mathbf{x}+\mathbf{r}) \\
& =E[f(\mathbf{x}) f(\mathbf{x}+\mathbf{r})],
\end{aligned}
$$

where $r=|\mathbf{r}|, f(\mathbf{x})$ is the observation at position $\mathbf{x}$ within the interferogram and where we assume that the average value of the noise is zero. Throughout this paper, boldface refers to vectors and matrices, while normal font refers to scalars. We also consider the structure function, which is typically defined [e.g., Williams et al., 1998; Hanssen, 2001; Jónsson, 2002] as the variance of the difference between two points separated by a distance $r$,

$$
S(r)=E\left[(f(\mathbf{x})-f(\mathbf{x}+\mathbf{r}))^{2}\right],
$$

such that

$$
C_{n}(r)=\sigma^{2}-S(r) / 2
$$

where $\sigma^{2}$ is the variance of noise within the image. Our assumption of stationary, isotropic noise implies that both $\mathbf{C}_{\mathbf{n}}$ and $\mathbf{S}$ are functions only of the scalar distance $r$.

[8] We examine two methods of calculating $\mathbf{C}_{\mathbf{n}}$ and $\mathbf{S}$ for a given interferogram. First, we explicitly compare all pairs of points within the interferogram, $\Phi$, by calculating the full autocorrelation matrix, A.

$$
\mathbf{A}_{d x, d y}=\frac{1}{n g} \sum_{k=d x}^{n x} \sum_{l=d y}^{n y} \Phi_{k, l} \Phi_{k-d x+1, l-d y+1},
$$

where $d x=1: n x, d y=1: n y, n x$ and $n y$ are the number of columns and rows in the interferogram, and $n g$ is the number of valid values within the overlapping region at each shift $(d x, d y)$. We keep track of the difference between each pair of points to form $\mathbf{S}$,

$$
\mathbf{S}_{d x, d y}=\frac{1}{n g} \sum_{k=d x}^{n x} \sum_{l=d y}^{n y}\left(\Phi_{k, l}-\Phi_{k-d x+1, l-d y+1}\right)^{2} .
$$

In general, interferogram noise is anisotropic and $\mathbf{A}$ varies with azimuth. Here, we neglect this anisotropy and assume that the azimuthal average of $\mathbf{A}$ versus distance approximates $C_{n}(r)$ (Figure 2).

[9] Our second technique for computing $\mathbf{C}_{\mathbf{n}}$ and $\mathbf{S}$ is a sample variogram, where we choose a large number of random pixel pairs within the interferogram, binned by distance (blue curve in Figure 2). For the example shown in Figures 1 and 2 we use an interferogram that has been subsampled down to less than a hundredth of its original size (i.e., in radar processing parlance, the interferogram has been looked down 10 times in range and 50 times in azimuth). For larger images, computation of the 

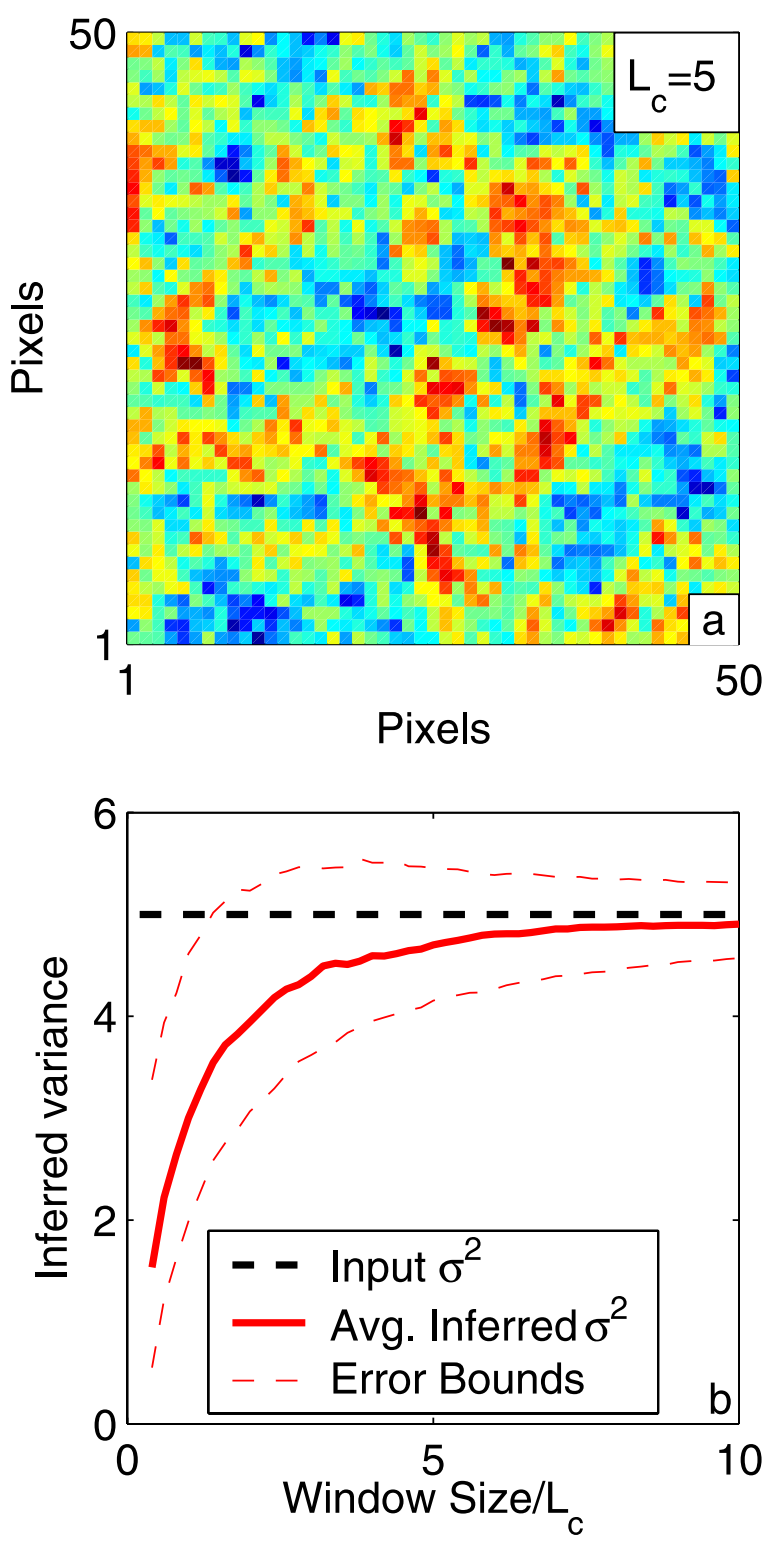

Figure 3. Dependence of inferred variance on window size. (a) Synthetic noise with covariance following an exponential decay with scale length, $L_{c}=5$ pixels. (b) Inferred variance of pixels versus window size. Note that the inferred variance approaches the input variance when the scale length of the noise is several times smaller than the window size.

autocorrelation and structure function using all possible pairs of points within the image becomes computationally prohibitive. Figure 2 indicates that it is instead sufficient to use a sample variogram, with an appropriately large number of random pairs of points.

[10] Theoretical work on atmospheric turbulence [e.g., Tatarski, 1961], GPS observations of tropospheric delay [e.g., Emardson et al., 2003], and previous studies of InSAR data [e.g., Goldstein, 1995; Zebker et al., 1997; Hanssen, 2001; Jónsson, 2002] all indicate that the spatial structure of the atmosphere exhibits power law behavior that is correlated over length scales of several hundreds of kilometers. However, we note some differences between the character of noise observed in InSAR data and that observed in GPS data. Three geometric considerations cause the covariance we observe within the interferogram to be different than the actual covariance between points across that particular time interval: the limited spatial scale of the interferogram, the fact that we observe phase delays relative to some arbitrary constant, and the fact that orbital errors introduce an uncertainty approximately corresponding to a quadratic ramp across the image. Many of the interferograms we examined have empirically estimated $C_{d}$ that decay exponentially over some scale length $L_{c}$.

[11] We explore how the three factors mentioned above affect the difference between inferred and actual covariances with several synthetic tests. We create synthetic correlated noise, $n_{c}$, from a predetermined covariance matrix, $\mathbf{C}_{\mathbf{d}}$, using the following relations:

$$
\begin{gathered}
C_{d}^{i j}=e^{-L^{i j} / L_{c}} \\
\mathbf{n}_{\mathbf{c}}=\mathbf{v u}^{1 / 2} \mathbf{n}_{\mathbf{n}},
\end{gathered}
$$

where $C_{d}^{i j}$ and $L$ are the covariance and distance between the $i$ th and $j$ th points, $n_{n}$ is uncorrelated noise, and $\mathbf{v}$ and $\mathbf{u}$ are the matrices of eigenvectors and eigenvalues of $\mathbf{C}_{\mathbf{d}}$, respectively. Not surprisingly, the covariance we infer for any particular set of noise depends in part on the spatial extent of the data set. The variance we estimate for subsets of pixels within synthetically generated correlated noise only approaches the input variance when the window size is several times larger than the correlation length scale of the noise (Figure 3). Furthermore, the covariance we infer from data with power law correlated noise changes when we begin by removing the mean or a quadratic ramp from the data (Figure 4). When we remove a quadratic ramp over an image approximately $100 \mathrm{~km}$ in extent, the remaining signal is only correlated on length scales of around $50 \mathrm{~km}$. The removal of the mean of the signal corresponds to the case where we believe we have good information on the orbital baselines and are confident that our interferogram characterizes the noise to within an arbitrary constant, whereas the removal of a 

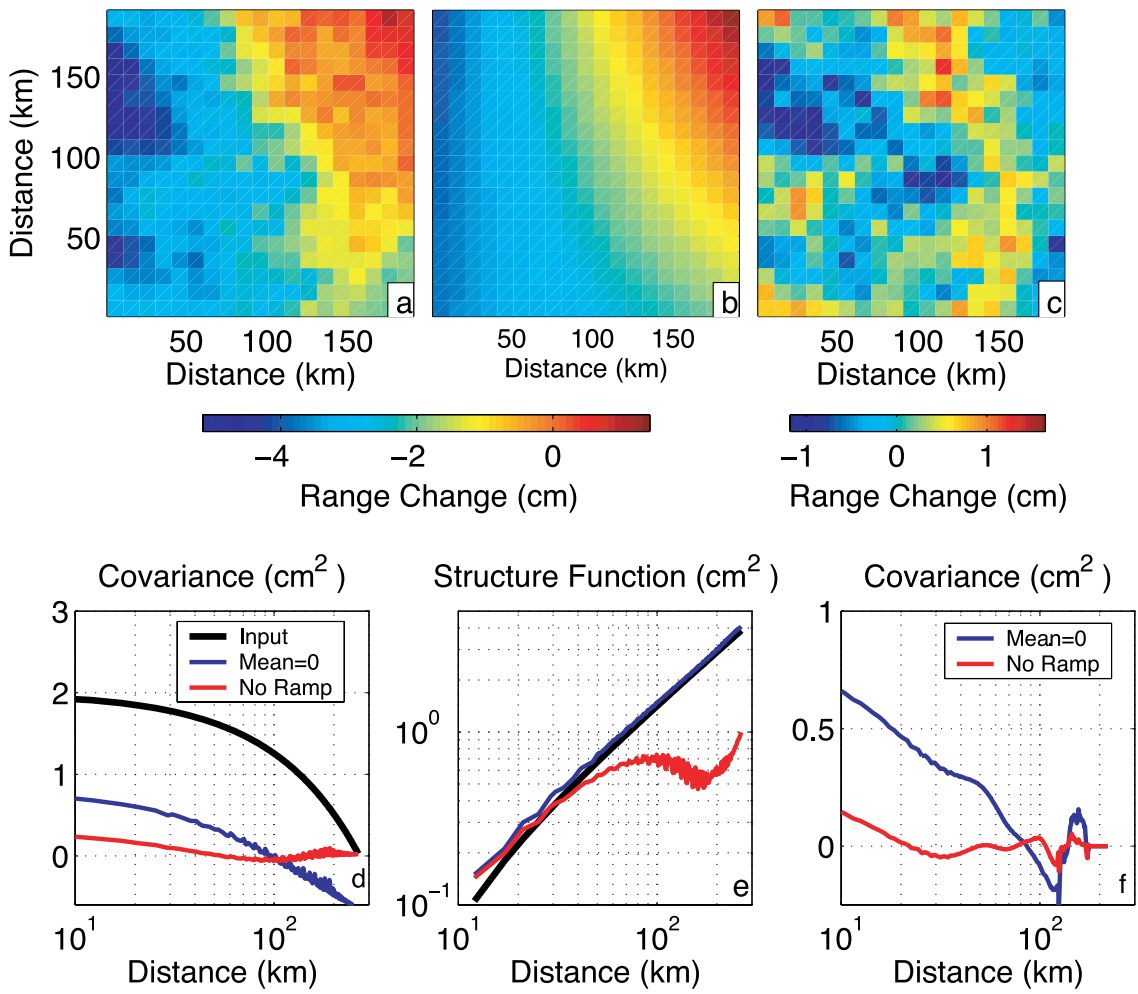

Figure 4. Effect of ramp removal on character of noise. (a) One realization of noise with power law structure function. (b) Best fit quadratic ramp to Figure 4a. (c) Remaining signal after ramp removal. (d) Input covariance (thick line) and inferred covariance for synthetic noise in Figure 4a after removal of mean (blue) and quadratic ramp (red). (e) Structure function for same scenario. (f) Covariance inferred for real data in Figure 1, with the removal of the mean and with and without the removal of the best fitting quadratic ramp.

quadratic corresponds to the more common case where we have inadequate knowledge of the satellite viewing geometries. Note that the blue and black lines overlay each other in Figure 4e, because the structure function is insensitive to the mean value of the noise. In Figure 4f, we show the inferred covariance for the real interferogram from Figure 1, both with and without the removal of the best-fitting ramp, analogous to the red and blue curves in Figures $4 \mathrm{~d}$ and $4 \mathrm{e}$. Both curves follow an exponential decay versus distance. Interferograms always include an arbitrary constant, so we can never reconstruct the true "input" signal (black curves in Figures 4d and 4e).

[12] We compare inferred noise covariances for several interferograms in the region surrounding the $1999 \mathrm{M}_{w}$ Hector Mine (California) earthquake with previous work based on simulating expected InSAR covariances by using a large ensemble of GPS observations of tropospheric delay in Southern California [Emardson et al., 2003]. In Figure 5a, we show the inferred spatial structure function for interferograms spanning different time intervals over the same location. Interferograms spanning the time of the Hector Mine earthquake are shown in black, and those using interseismic interferograms are shown in gray. When the interferogram spans the earthquake, we remove a model of the coseismic deformation before we examine the noise structure. Tests where we removed data proximal to the fault resulted in indistinguishable covariance functions, so we believe that removing a coseismic model does not significantly bias our estimate of the noise covariance. The vertical scatter of the InSAR curves reflects the fact that each represents independent realizations of the atmospheric noise. The similarity in the spatial scale of the noise between coseismic and interseismic interferograms $(\sim 10 \mathrm{~km})$ indicates that our estimate of the noise structure is insensitive to our method for removing the coseismic signal.

[13] The disagreement in functional form between our observations and the GPS data at distances greater than $10^{2} \mathrm{~km}$ results from the fact that we perform a series of filtering operations on the InSAR data during the formation of the interferogram, including the absorption of a quadratic ramp by an interferometric baseline reestimation proce- 

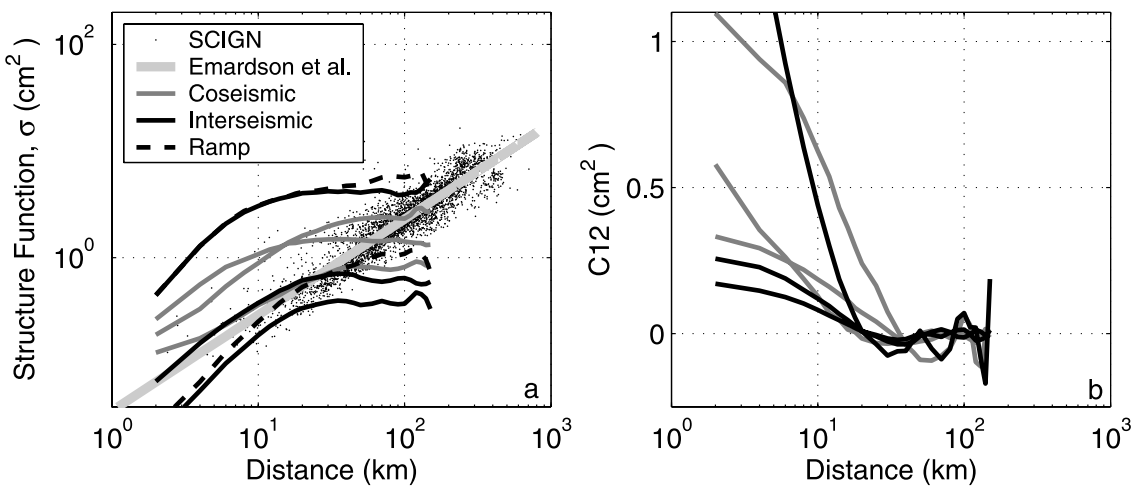

Figure 5. (a) Spatial structure function versus distance for interferograms spanning the Hector Mine event (black), and interferograms spanning time periods before or after the earthquake (gray). Dashed lines indicate the structure function for the interferogram before a quadratic ramp was removed. GPS data (small dots) are from the Southern California Integrated GPS Network (SCIGN) (adapted from Emardson et al. [2003]). The thick grey line indicates the power law fit from Emardson et al. [2003]. (b) Covariance versus distance for the coseismic and interseismic interferograms from Figure 5a.

dure mentioned above. The dashed curves indicate the structure function we would infer if we did not remove a ramp from the final interferogram, which corresponds to the blue curve in Figure 4, where we only remove the mean from the data. One benefit of empirical covariance and structure functions compared with theoretical calculations of noise structure is that we implicitly include the effect of the filtering, averaging, and DEM errors that are part of the interferogram processing.

\section{Resolution-Based Resampling}

[14] Inversion of interferograms at full resolution can require evaluating the forward problem at over $10^{7}$ points, which is computationally expensive. This expense is magnified for nonlinear problems where we adopt Monte Carlo optimization techniques. We can reduce the number of data points without losing significant information by taking appropriate spatial averages. In averaging the data we should take advantage of the fact that individual neighboring pixels far from the deformation source contribute approximately identical information to the inversion.

[15] In this section we describe an algorithm for choosing an appropriate set of spatial averages, and compare our result with previous methods for spatially resampling interferograms. We define a set of $n$ orthogonal boxcar functions, $\mathbf{a}_{\mathbf{i}}$, such that

$$
\begin{gathered}
\sum_{x} a_{i}(x) a_{j}(x)=0 \\
D_{i}=\sum_{x} a_{i}(x) d(x),
\end{gathered}
$$

where $x$ is a spatial coordinate covering the interferogram, $d$ are the original, full resolution data, and $D_{i}$ are the resulting individual resampled data points. We infer a source model, $\mathbf{m}$, from a given $\mathbf{D}$ using the forward relation

$$
\mathbf{G m}=\mathbf{D},
$$

where $\mathbf{G}$ is the design matrix of Green's functions. A great variety of inverse methods exists for finding the family of slip models that solve equation (10) [e.g., Parker, 1977; Tarantola and Valette, 1982; Menke, 1989]. The goal of any resampling algorithm is to reduce the size of $\mathbf{G}$ and D, which control the number of computationally expensive forward models that we have to compute, without significantly degrading the information about $\mathbf{m}$ contained in the inversion. The character of $\mathbf{G}$ controls the shape of the optimal distribution of $\mathbf{D}$, since we generally would like to have the highest density of data points close to the source of deformation.

[16] Previous techniques for resampling InSAR data include uniform sampling, in which the $\mathbf{a}_{\mathbf{i}}$ form a regular grid across the image [e.g., Pritchard et al., 2002], and resampling techniques based on the spatial complexity of $\mathbf{d}$. Examples of algorithms that sample the data according to their spatial variance is given by Simons et al., [2002] and Jónsson et al. [2002]. Both methods begin by dividing the data into quadrants and subdividing each quadrant until the variance of the data within each smaller quadrant is below a preset threshold. The two techniques differ in the function removed from each sub-quadrant before estimating the variance. Jónsson et al. [2002] remove the local 


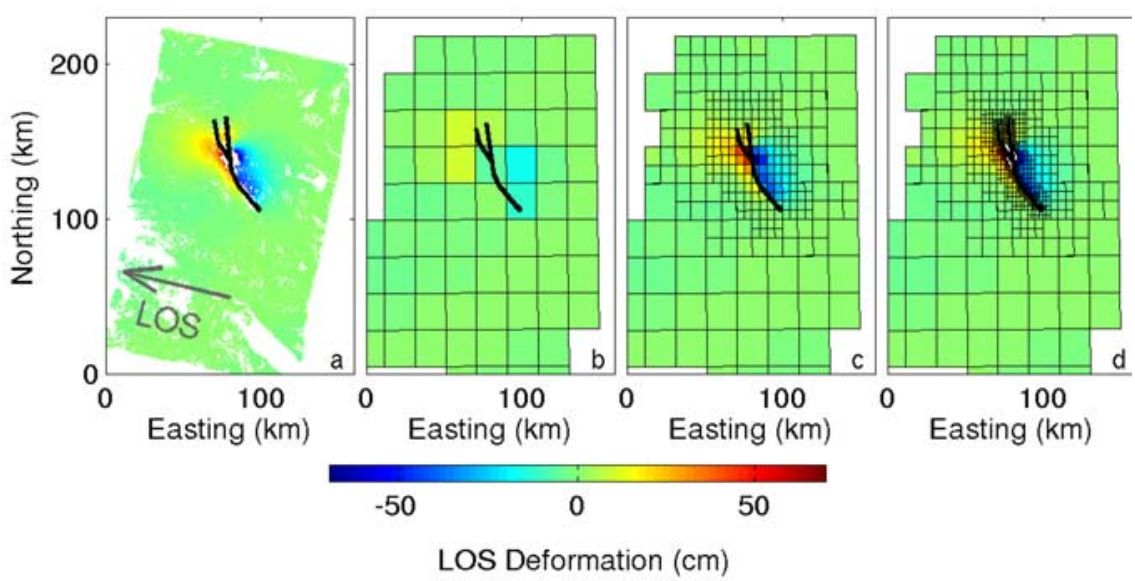

Figure 6. (a) Input unwrapped interferogram for Hector Mine earthquake: Track 127, Frame 2907, spanning 13 January 1999 to 20 October 1999. Color scale indicates the LOS change toward or away from the satellite in the direction indicated by the arrow. Black lines indicate the fault plane. $(b-d)$ Iterations 1,3 , and 6 of the R-based resampling algorithm, with color indicating the average LOS value for all non-NAN points contained within each box.

mean, while Simons et al. [2002] remove the bestfitting local bilinear ramp. The Simons et al. [2002] algorithm is sensitive only to the curvature of the signal, whereas the sampling density of the algorithm used by Jónsson et al. [2002] also depends on the local slope, and therefore the absolute amplitude, of the deformation field.

[17] Uniform sampling is not ideal for deformation sources which approach or break the surface, because it may require an unnecessarily large number of data points in the inversion to achieve dense sampling near the source where the data has the most detailed structure. For subduction zone events or other deep sources of deformation [e.g., Pritchard et al. 2002], uniform sampling can be a reasonable approach. Both the Simons et al. [2002] and Jónsson et al. [2002] algorithms may produce high densities of data far from the deformation source in the presence of small-scale perturbations to the data from noise, decorrelation, or phaseunwrapping errors. These concentrations of points decrease inversion efficiency without significantly improving the inferred model.

[18] We introduce an algorithm that uses the design of the inverse problem to determine the optimal data sampling density. We examine the data resolution matrix for a given set of $\mathbf{a}_{\mathbf{i}}$, beginning with a coarse uniform sampling. If any particular $\mathbf{a}_{\mathbf{i}}$ is resolved above a prescribed threshold, we partition it into four smaller regions and re-examine the new data resolution. Our data resolution-based (Rbased) sampling algorithm is as follows: (1) define the source parameterization (e.g., fault plane geometry); (2) begin with a coarse grid of $\mathbf{a}_{\mathbf{i}}$ and form $D_{i}$; (3) compute Green's functions; (4) form generalized inverse, $\mathbf{G}^{-\mathbf{g}}=\left(\mathbf{G G}^{\prime}\right)^{-1} \mathbf{G}^{\prime}$ [e.g., Menke, 1989]; (5) calculate data resolution matrix, $\mathbf{N}=\mathbf{G G}^{-\mathbf{g}}$; (6) find all $D_{i}$ where the diagonal of $\mathbf{N}$ is above preset threshold; (7) subdivide points from step 6 into four parts, forming new $\mathbf{a}_{\mathbf{i}}$; and (8) repeat steps 3-7 until all $D_{i}$ are just below threshold. Here $\mathbf{G}$ is the matrix of Green's functions augmented by a finite difference approximation of the Laplacian smoothing matrix [e.g., Menke, 1989]. Our two tuning parameters are the resolution threshold (between 0 and 1) and the weighting of smoothing included in G. For a reasonably small size of fault patch, we must apply some smoothing to avoid numerical difficulties in the inversion. If the resolution threshold is close to zero or if the smoothing is small, then the final number of $D_{i}, n$, will be higher. Currently, the choice of tuning parameters is somewhat arbitrary and is driven simply by the desire to have a low number of points (so that the computational cost is reasonable) that are concentrated near the fault. In our implementation, we also remove samples corresponding to regions that cross the fault. The number of data points in each box does not simply scale with box size, since some boxes contain decorrelated regions. We illustrate how the sampling evolves over several steps using inSAR data for the $1999 M_{w} 7.1$ Hector Mine earthquake that we will examine further in section 4 (Figure 6).

[19] Our inversion would not be complete without a data covariance matrix associated with our 

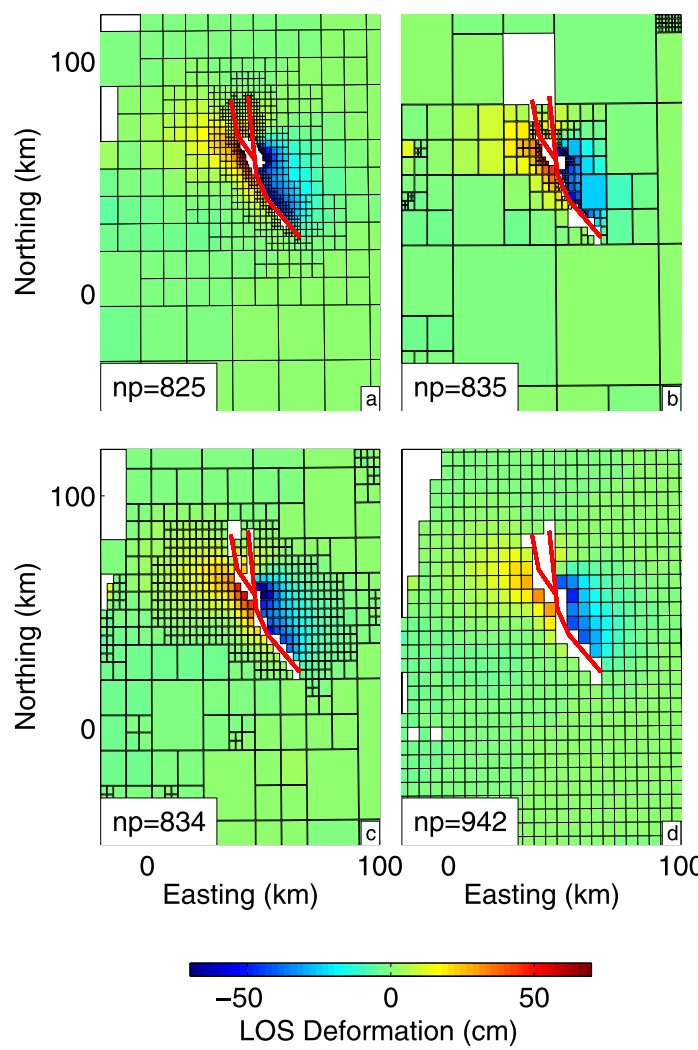

Figure 7. Resampling results using each algorithm discussed in the text. (a) R-based algorithm. (b) Simons et al. [2002] algorithm. (c) Jónsson et al. [2002] algorithm. (d) Uniform sampling.

resampled $D_{i}, \mathbf{C}_{\mathbf{D}}$, allowing us to estimate the covariance matrix for the final inferred model. Recognizing that each $D_{i}$ is a sum over a subset of $d$, we express the covariance between any $D_{i}$ and $D_{j}$ as

$$
\operatorname{Cov}\left(D_{i}, D_{j}\right)=\operatorname{Cov}\left(\frac{1}{n_{i}} \sum_{x=a_{i}} d(x), \frac{1}{n_{j}} \sum_{x=a_{j}} d(x)\right)
$$

or

$$
\operatorname{Cov}\left(D_{i}, D_{j}\right)=\frac{1}{n_{i} n_{j}} \sum_{x_{i}=a_{i}} \sum_{x_{j}=a_{j}} \operatorname{Cov}\left(d\left(x_{i}\right) d\left(x_{j}\right)\right) .
$$

The covariance between any two $D_{i}$ is the sum of the covariance between all points included in their averages, as determined in section 2. Likewise, the variance of any individual $D_{i}$ accounts for all the data points averaged by $\mathbf{a}_{\mathbf{i}}$.

[20] When we perform an inversion of geodetic data (InSAR or GPS), we transform our system of equations by premultiplying with $\mathbf{P}=\mathbf{Q}^{-1}$, where $\mathbf{Q}$ is the Cholesky factorization of $\mathbf{C}_{\mathbf{d}}$ ( or $\left.\mathbf{C}_{\mathbf{D}}\right)$ such that $\mathbf{Q Q} \mathbf{Q}^{T}=\mathbf{C}_{\mathbf{d}}$. If we use the correct $\mathbf{C}_{\mathbf{d}}$, this transform results in reweighted data with unit variance [e.g., Harris and Segall, 1987; Segall and Harris, 1987].

\section{Example 1: A Large Shallow Strike- Slip Earthquake}

[21] We demonstrate the behavior of our resampling algorithm on inversions for subsurface fault slip using synthetic data similar to the InSAR data spanning the 1999 Hector Mine earthquake $\left(\mathrm{M}_{w}\right.$ 7.1). We consider only synthetic scenarios because we can separate out the effects of noise and data distribution from model errors in fault plane geometry and elastic models of the crust. We use a fault plane geometry modified from Simons et al. [2002], and do not include correlated noise in this particular example.

[22] To make our synthetic examples similar to a real-world scenario, we generate synthetic data using an input slip model loosely based on one inferred from the real InSAR data (Figure 6a). The slip model inferred from real data is already a smoothed representation of the actual slip distribution, so we add Gaussian peaks in slip at several locations on the fault plane to restore a more plausible complexity to the input slip distribution. We add random noise to the synthetic data with variance $\sigma^{2}=1 \mathrm{~cm}$. Because this example is for demonstration purposes, we add a slightly higher level of random noise to several regions around the outside of the interferogram to simulate DEM and unwrapping errors and to show how some of the resampling algorithms can concentrate sampling far from the areas of interest.

[23] To compare our algorithm with the other styles of resampling, we tune each resampling technique so that it produces approximately the same number of data points (Figure 7). We apply each resampling algorithm to the full data set, using the same synthetic noise in each case. We remove averaging boxes that cross the fault, since in general we will not have the capability to model near-fault processes. We then compute Green's functions for the resulting resampled data geometry and compare the inferred slip model for each set of $D$. We use the ${ }_{j} R_{i}$-criterion (R. Lohman and M. Simons, Inferring fault slip from surface deformation using a spatially variable regularization scheme, submitted to Geophysical Journal International, 2004) to pick the optimal value of smoothing in each case. 

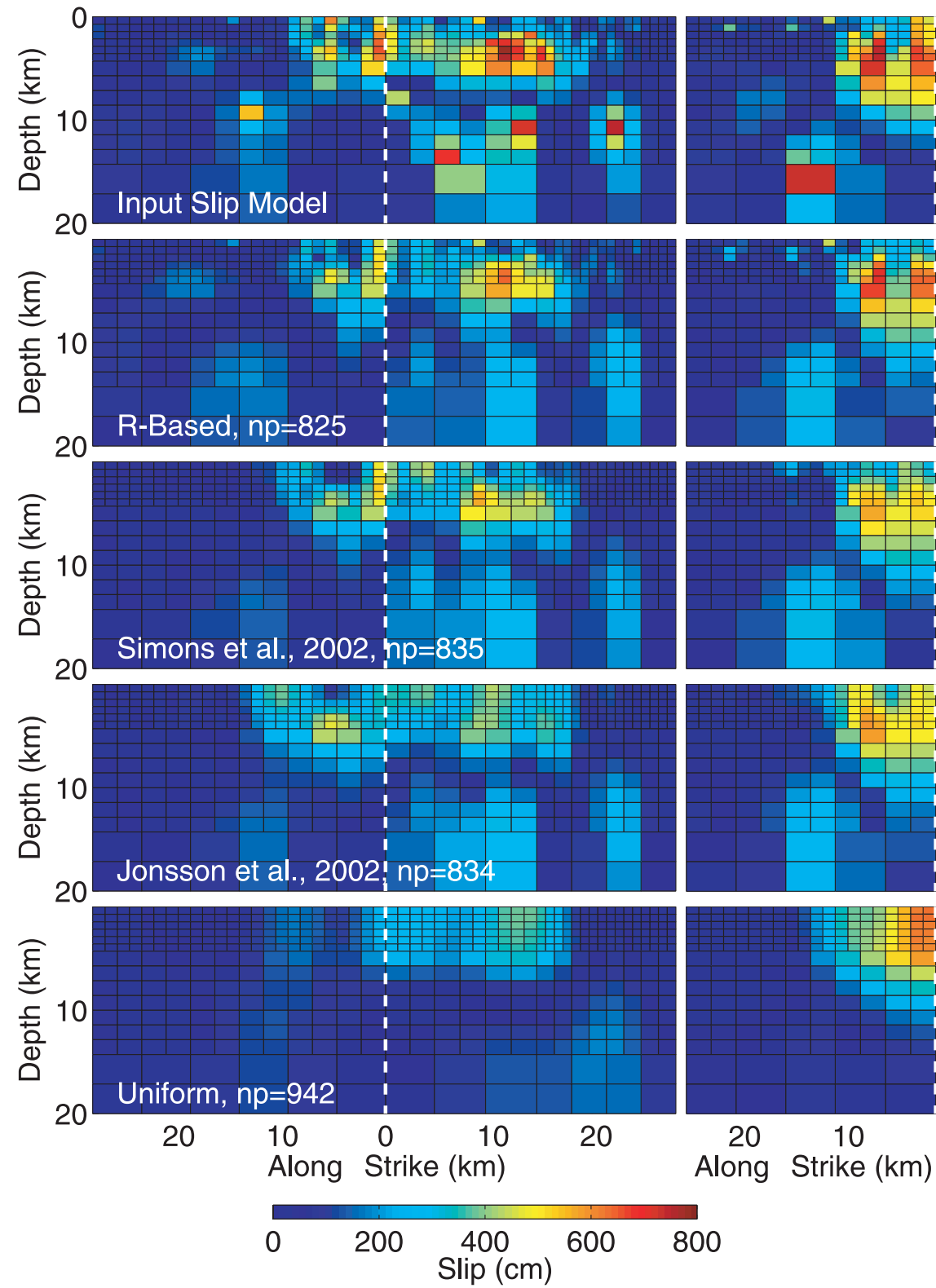

Figure 8. Input slip model and comparison of slip models inferred from R-based algorithm, Simons et al. [2002], Jónsson et al. [2002], and uniform sampling. The abscissa corresponds to the horizontal distance from the "Y" intersection (indicated by dashed white lines) of the Hector Mine fault plane (Figure 6). Left and right panels correspond to the east and west sides of the "Y," respectively. Note that inferred slip is similar for all inversions, but that R-based resampling better distinguishes between shallow slip features.

[24] The uniform algorithm is clearly not ideal in this example, as is shown by the lower resolution of the slip model in Figure 8. The Simons et al. [2002] and Jónsson et al. [2002] algorithms have higher concentrations of data near the fault, but there are also clusters of data points associated with the noisy regions on the edges of the interferogram. The Simons et al. [2002] method shows less vertical smearing than the Jónsson et al. [2002] approach for this particular choice of tuning parameters. Even though they use slightly more data points, the Simons et al. [2002] and Jónsson et al. [2002] slip models retain fewer of the features in the original slip model than does the R-based slip model.

[25] We note that the R-based algorithm will concentrate sampling around the fault plane even if there is not a large variation in the actual data values associated with a given part of the fault trace. Therefore we can resolve regions of the fault plane that did not slip during the earthquake, a 


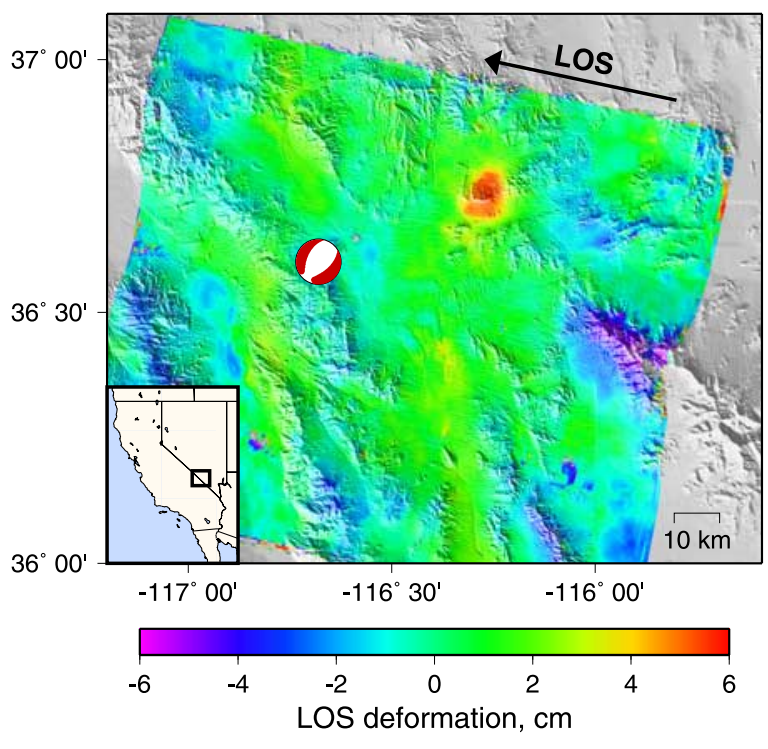

Figure 9. Interferogram (14 May 1993 to 24 April 1992) spanning 29 June 1992 Little Skull Mountain earthquake draped over shaded relief map, with Harvard CMT focal mechanism (red) and the satellite-to-ground LOS vector (black).

feature that can be as important as finding the peaks in the slip distribution. The large data gap at the north end of the fault in Figure $7 \mathrm{~b}$ results from the fact that the data-based Simons et al. [2002] algorithm did not sample the ends of the fault well, so that when we removed all boxes crossing the fault plane, we removed a box that sampled a large area.

\section{Example 2: A Small Shallow Earthquake}

[26] We show how InSAR data can constrain the location of shallow earthquakes to within a few kilometers even when the event is fairly small by teleseismic standards. Precise earthquake locations can be used as input to seismic structural studies, analysis of styles of continental deformation, and can help calibrate earthquake location techniques otherwise based only on seismology. The ability to locate small, shallow seismicity is an important part of the Comprehensive Nuclear Test Ban Treaty (CTBT) program. Equally important to all of these applications is our ability to place confidence limits on our inferred location for a given event. In this section, we show how the use of Monte Carlo methods combined with inaccurate knowledge of the noise covariance can lead to incorrect error bounds on the inferred earthquake location.
[27] We follow up on a previous work [Lohman et al., 2002] where we used InSAR and broadband seismic waveform data to estimate source parameters of the 29 June $1992 \mathrm{M}_{s} 5.4$ Little Skull Mountain earthquake (Figure 9). In our previous work [Lohman et al., 2002], we did not use the full noise covariance matrix to estimate error bounds on our best-fitting model parameters. Here we demonstrate the impact of using the correct covariance on the inferred error bounds. We infer the best-fitting hypocenter, fault plane area, and fault slip using the Neighborhood Algorithm [Sambridge, 1998]. Since the existing data set is limited to observations from only one line-of-sight (LOS) direction, we have low sensitivity to the mechanism of this event. We fix the strike, dip, and rake to the values determined from seismic data by Lohman et al. [2002] for our estimation of the bestfitting parameters, but allow the mechanism to vary when we perform the error analysis described below.

[28] In order to determine the error bounds we associated with our location, we perform a Monte Carlo sensitivity analysis. We create a large number $(>500)$ of independent sets of synthetic noise drawn from the covariance matrix of the noise as estimated from the original interferogram, and add the best-fitting synthetic model from our nonlinear inversion. For each realization of the noise, we invert for the new best-fitting earthquake parameters (hypocenter, mechanism, fault plane area, and fault slip) using the Neighborhood Algorithm. In Figure 10, we show the result of the inversion of each synthetic data set as a small red dot, with $1 \sigma$ confidence ellipse (red) derived from the distribution of synthetic models. If we generate noise using only the diagonal of the covariance matrix, neglecting any spatial correlation of the noise, we underestimate our errors by nearly a factor of 2 .

\section{Conclusions}

[29] By making the assumption that the noise is spatially stationary and isotropic, we ignore the fact that interferograms often contain azimuthally dependent noise due to the correlation between topographic structures and atmospheric water vapor (Figure 9a). Our estimate of the noise covariance is, therefore, not adequate for statistical methods such as the maximum likelihood estimation of the optimal smoothing parameter, where the exact character of the noise must be known. However, the treatment of noise covariance presented here improves over the complete neglect of 

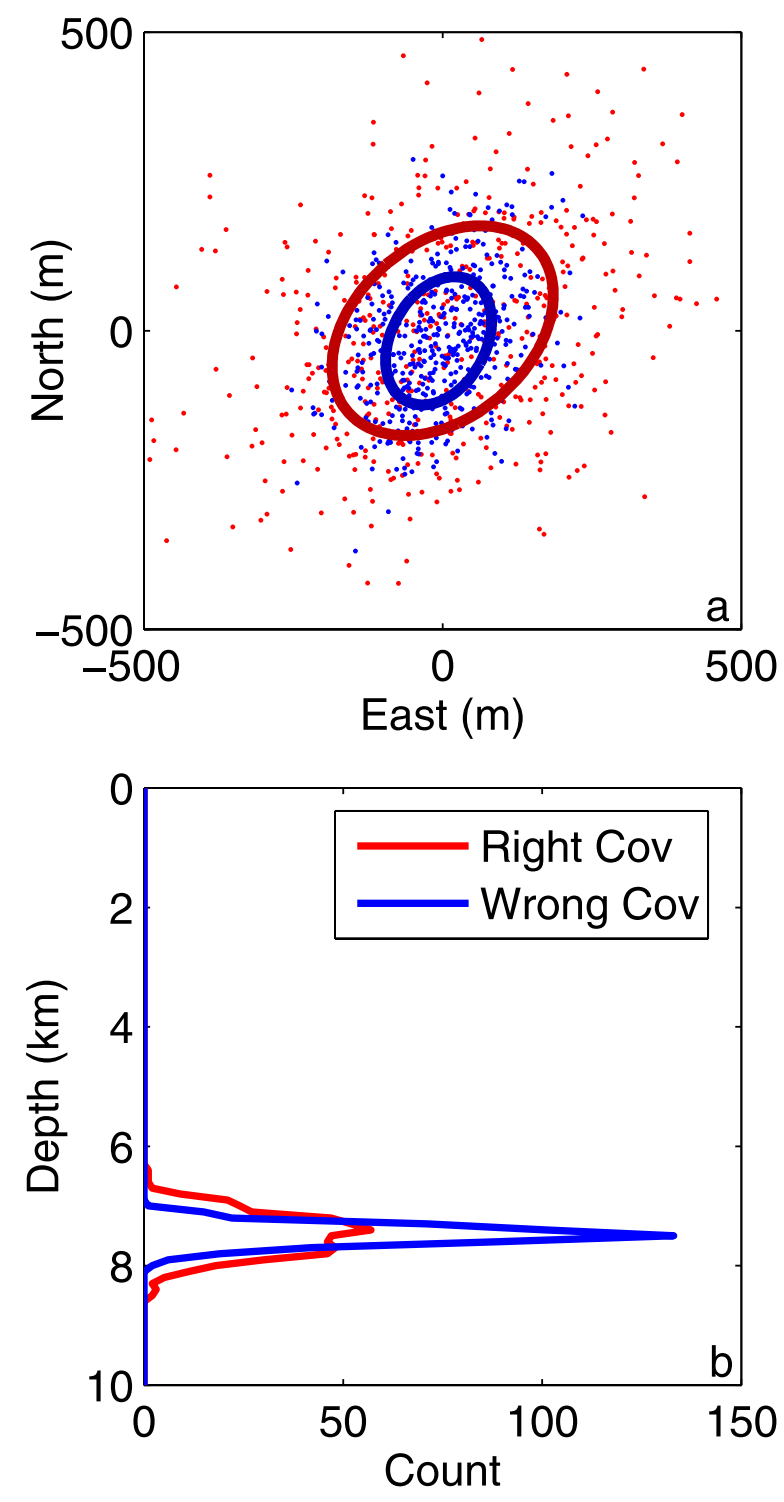

Figure 10. Comparison of confidence intervals for correct and incorrect noise covariance matrices, $C_{n}$. (a) Each dot indicates the inferred map view location for one independent realization of the noise, drawn from either the correct (red) or incorrect (blue) covariance matrix. Solid lines correspond to the $1 \sigma$ confidence intervals derived from the Monte Carlo tests. (b) Histogram of inferred depths for each of the 500 Monte Carlo tests. Note how the confidence intervals for each quantity are tighter if we use the incorrect covariance matrix.

spatial correlation of noise between individual data points and can be of great value in Monte Carlo methods [e.g., Wright et al., 2003, 2004]. We find that the covariance structure inferred directly from the InSAR data will be different than the actual covariance between data points at a given distance, because of the limited spatial aperture of an InSAR scene and the tradeoff between spatial covariance and satellite orbital errors. We note that a combination of error bounds on the satellite orbits and dense GPS observations at the time of the InSAR data acquisitions may potentially be used to reduce this tradeoff.

[30] We conclude that the R-based resampling is a robust technique that provides dense sampling in regions where the data has the most relevant structure for the deformation source of interest, while circumventing problems that other resampling techniques have when applied to noisy data. We note that the R-based resampling may miss deformation sources that are not included in the model used to resample the data, such as triggered slip on nearby faults during an earthquake, but that these oversights can be detected by examining the residual with the full data set at the end of the inversion. In this paper we only considered InSAR data, but our resampling algorithm could be easily applied to other forms of spatially dense geodetic imagery such as pixel tracking using optical or radar images [e.g., Michel and Avouac, 2002; Simons et al., 2002]. We also demonstrate the need to include the spatial correlation of InSAR noise in inversions for earthquake parameters, especially when the signal of interest has approximately the same spatial scale as the noise. Combining these two techniques helps optimize the amount of information we can extract from InSAR data.

\section{Acknowledgments}

[31] We acknowledge the helpful comments of the editor and two anonymous reviewers. R. Lohman is partially supported by a NASA New Investigator Program grant award to M. Simons. Contribution 9097, Division of Geological and Planetary Sciences, California Institute of Technology, Pasadena, California.

\section{References}

Emardson, T. R., M. Simons, and F. H. Webb (2003), Neutral atmospheric delay in interferometric synthetic aperture radar applications: Statistical description and mitigation, J. Geophys. Res., 108(B5), 2231, doi:10.1029/ 2002JB001781.

Goldstein, R. (1995), Atmospheric limitations to repeat-track radar interferometry, Geophys. Res. Lett., 22, 2517-2520.

Hanssen, R. A. (2001), Radar Interferometry: Data Interpretation and Error Analysis, Springer, New York.

Harris, R. A., and P. Segall (1987), Detection of a locked zone at depth on the Parkfield, California, segment of the San Andreas Fault, J. Geophys. Res., 92, 7945-7962.

Jónsson, S. (2002), Modeling volcano and earthquake deformation from satellite radar interferometric observations, Ph.D. thesis, Stanford Univ., Stanford, Calif. 
Jónsson, S., H. Zebker, P. Segall, and F. Amelung (2002), Fault slip distribution of the $1999 \mathrm{~m}_{w}$ 7.1 Hector Mine, California, earthquake, estimated from satellite radar and GPS measurements, Bull. Seismol. Soc. Am., 92, 1377-1389.

Lohman, R., M. Simons, and B. Savage (2002), Location and mechanism of the Little Skull Mountain earthquake as constrained by satellite radar interferometry and seismic waveform modeling, J. Geophys. Res., 107(B6), 2118 , doi:10.1029/2001JB000627.

Menke, W. (1989), Geophysical Data Analysis: Discrete Inverse Theory, Int. Geophys. Ser., vol. 45, rev. ed., Elsevier, New York.

Michel, R., and J. P. Avouac (2002), Deformation due to the 17 August Izmit earthquake measured from spot images, J. Geophys. Res., 107(B4), 2062, doi:10.1029/ 2000JB000102.

Parker, R. L. (1977), Understanding inverse theory, Annu. Rev. Earth Planet. Sci., 5, 35-64.

Pritchard, M. E., M. Simons, P. A. Rosen, S. Hensley, and F. H. Webb (2002), Co-seismic slip from the 1995 July 30 $\mathrm{Mw}=8.1$ Antofagasta, Chile, earthquake as constrained by InSAR and GPS observations, Geophys. J. Int., 150, 362-376.

Sambridge, M. (1998), Geophysical inversion with a neighborhood algorithm: I. Searching a parameter space, Geophys. J. Int., 138, 479-494.
Segall, P., and R. Harris (1987), Earthquake deformation cycle on the San Andreas Fault near Parkfield, California, J. Geophys. Res., 92, 10,511-10,525.

Simons, M., Y. Fialko, and L. Rivera (2002), Coseismic deformation from the $1999 \mathrm{Mw} 7.1$ Hector Mine, California, earthquake as inferred from InSAR and GPS observations, Bull. Seismol. Soc. Am., 92, 1390-1402.

Tarantola, A., and B. Valette (1982), Inverse problems $=$ quest for information, J. Geophys., 50, 159-170.

Tatarski, V. L. (1961), Wave Propagation in a Turbulent Medium, McGraw-Hill, New York.

Williams, S., Y. Bock, and P. Fang (1998), Integrated satellite interferometry: Tropospheric noise, GPS estimates, and implication for interferometric synthetic aperture radar products, J. Geophys. Res., 103, 27,051-27,067.

Wright, T. J., Z. Lu, and C. Wicks (2003), Source model for the $\mathrm{M}_{w}$ 6.7, 23 October 2002, Nenana Mountain Earthquake (Alaska) from InSAR, Geophys. Res. Lett., 30(18), 1974, doi:10.1029/2003GL018014.

Wright, T. J., B. E. Parsons, and Z. Lu (2004), Toward mapping surface deformation in three dimensions using InSAR, Geophys. Res. Lett., 31, L01607, doi:10.1029/2003GL018827.

Zebker, H. A., P. A. Rosen, and S. Hensley (1997), Atmospheric effects in interferometric synthetic aperture radar surface deformation and topographic maps, J. Geophys. Res., 102, 7547-7563. 